\title{
Rate Region of Multi-channel Mesh Data Networks with Rate Control
}

\author{
Nidhi Hegde \\ Orange Labs R \& D \\ France
}

\author{
Alexandre Proutiere \\ Microsoft Research \\ Cambridge, UK
}

\begin{abstract}
Mesh networking has recently been advocated as an efficient and low-cost approach for providing high speed access to the Internet. Before such networks are able to support high-bandwidth data transfers in reality, a number of challenges must be addressed including routing, channel and radio interface assignments, radio resource management, and MAC scheduling. Our focus in this paper is to provide guidelines on the design of optimal radio resource management and distributed MAC scheduling algorithms. To this aim, we develop a general analytical model to characterize the performance of such networks. This task comprises many major difficulties: analyzing the behavior of distributed MAC schedulers in multi-hop networks, accounting for the interaction of links through interference and quantifying the impact of underlying congestion control algorithms. We present a unified approach partially based on decoupling arguments to address these challenges. Specifically, we provide approximations of the rate region of the network. Based on the derived results, we can propose a set of design rules for mesh multi-channel networks. For example, we investigate how to parametrize the coverage of the RTS/CTS signalling messages in IEEE 802.11-based networks.
\end{abstract}

\section{INTRODUCTION}

Mesh networking has been advocated as an efficient and low-cost approach for providing high speed access to the Internet. This architecture is considered to be well-suited for providing high-rate last mile connectivity, and as an alternative to wired LANs in offices [1]. Some cities have already deployed mesh multi-hop networks, to offer ubiquitous Internet wireless access across the city (see [2] and references therein).

In mesh networks, as opposed to ad-hoc networks, nodes are assumed to be relatively fixed, so that topology changes are not frequent. While this significantly simplifies the design and the practical implementation of certain mechanisms, such as the routing scheme, there remain several important challenges to be addressed before mesh networks are able to support highbandwidth data transfers or other high-rate applications [3].

Popular deployments of multi-hop networks are based on the IEEE $802.11 \mathrm{a} / \mathrm{b} / \mathrm{g}$ [4], and IEEE 802.16 [5] standards. These technologies offer multiple orthogonal channels (e.g., IEEE $802.11 \mathrm{~b}$ has 3 orthogonal channels, whereas in the IEEE 802.11a standard, up to 12 orthogonal channels can be used). Having multiple channels may alleviate one of the main difficulties in designing high-capacity wireless networks, namely interference. If the network can use many channels, one can assign the various channels to different links so that two links using the same channel are far apart, and cause little interference to each other. If the number of channels is limited relative to the network size, then the channel assignment cannot completely remove interference. Furthermore, to fully exploit the use of several channels, the nodes should possess several radio interfaces, or Network Interface Cards (NICs). Today, nodes typically have one to three NICs. An important issue then is the design of efficient channel and NIC assignment so as to maximize the network capacity. This design may also be jointly performed with that of the routing scheme and of the MAC protocol. The literature addressing this very complex issue is quite diverse. Some authors propose practical assignment and/or routing algorithms, see [2], [6][9]. Algorithms are defined for various scenarios depending on the number of channels and on the number of NICs available at each node. Other authors rather aim at identifying the optimal centralized algorithms leading to the greatest rate region of the network, i.e., the greatest set of the rates at which flows can be simultaneously transmitted on different routes. Even with simple assumptions on the use of channels and NICs and in case of networks with a limited number of nodes and routes, this problem turns out to be quite complex [10], [11].

Closely related to the channel assignment and routing problems is another important issue. interference management. This issue is especially challenging when the number of channels available is limited. Interference at a given receiver can be handled in two ways. The first is to control the transmission powers of the neighboring links. A radical way of controlling power is to impose that the neighboring links stay silent during the transmission of a given node. This is in fact the idea behind the RTS/CTS signaling used in IEEE 802.11 networks: the transmitter first ensures that the links interfering at the receiver remain silent, and then transmits at full power to the receiver. We call this the max power principle. One can however imagine more elaborate ways of tuning power, see e.g. [12]. The second way of handling interference is to use rate control, that is to adapt the transmission rate to the level of interference. This can be done before the packet transmission using channel prediction, or even during the transmission using advanced coding techniques such as incremental redundancy. There is no real consensus on the optimal way of handling interference in mesh networks with distributed resource allocation, although preliminary results indicate that the max power principle is optimal when rate control is possible [13]. This means that the strongest interferers are shut down, whereas the impact of the 
other interferers is controlled using rate control mechanisms).

The design of mesh multi-channel networks therefore seems extremely complicated in view of all the possible choices: channel/NIC assignment, routing, distributed MAC protocols, rate or power management. This clearly highlights the need to develop a robust performance model for such networks, that may serve as a guide towards the right design decisions. Our contributions here fall under both these objectives, performance evaluation and formulation of good design principles. In general, our aim is to derive a complete, yet usable analytical model that is able to predict the performance as perceived by users in multi-hop multi-channel wireless networks. A first step towards this aim is to quantify the performance assuming a fixed population of active data flows. In this paper, we derive an approximation of the rate region of the network for any fixed population of active flows. The proposed approach can be applied to various types of distributed MAC protocols. It allows us to model the interaction of the different links through interference. It also accounts for the basic characteristics of congestion control protocols (TCP), and also for the nonnegligible impact of the traffic generated by TCP acknowledgments ${ }^{1}$.

The proposed performance model can provide useful insight into the design of mesh networks. In the present paper, we provide an immediate application of our model: we provide an optimal parametrization of the RTS/CTS scheme in IEEE 802.11-based networks, that is, we identify the optimal range of coverage of the RTS/CTS signalling messages.

The rest of the paper is organized as follows. In the next section, we present the network model and the traffic assumptions. In Section II-E, we introduce the notion of rate region in data networks. Section III constitutes the main contribution of the paper and presents the analytical model providing an approximation of the rate region of mesh networks. In Section IV, we apply the model to investigate the optimal parametrization of the range of coverage of RTS/CTS messages in IEEE 802.11-based networks. In Section V we propose a numerical example to illustrate our analytical results.

\section{NETWORK / TRAFFIC MODELS AND RATE REGION}

In this section we propose a model for multi-hop multichannel wireless networks. We first describe the network topology, how links interact through interference, and how radio resources are shared among active links. We then specify the characteristics of traffic demand. Finally, we introduce the notion of rate region of the network that will serve to analyze the efficiency of the network mechanisms.

\section{A. Network topology, channel and NIC assignment}

We consider a network consisting of a set $\mathcal{N}$ of nodes and a set $\mathcal{L}$ of links between these nodes. A set $\mathcal{C}$ of channels are available for the links. A node belongs to a link $l$ if it is either its source $s(l)$ or its destination $d(l)$. We denote by $l^{-}$the reverse link corresponding to $l$, i.e., $s\left(l^{-}\right)=d(l)$ and

\footnotetext{
${ }^{1}$ in [14], the authors demonstrate that in 802.11 b networks, the transmission of TCP acks reduces by up to $40 \%$ the effective network capacity.
}

$d\left(l^{-}\right)=s(l)$. At least one channel is allocated to links $l$ and $l^{-}$; denote by $c(l)$ this channel. Also this node dedicates at least one of its NICs to these links. We further assume that the channel assignment is fixed.

\section{B. Rate control and radio propagation model}

The instantaneous capacity of a link is a function of the transmission power, the attenuation between the transmitter and the receiver, and the interference of other links transmitting on the same channel. We assume here that there is an explicit rate control algorithm, such as incremental redundancy, rapidly adapting the rate of transmission to the actual radio conditions at the receiver. In the following, we denote by $R_{l, \mathcal{A}}$ the rate of link $l$ when the set of active links is given by $\mathcal{A} \subset \mathcal{L}$. Note that we do not specify the transmission power of the different links: this implicitly means that all nodes operate at full power when transmitting.

We will use the following radio propagation model. We assume that the link rate is well approximated by Shannon formula (up to a multiplicative factor):

$$
R_{l, \mathcal{A}}=W \times \log _{2}(1+\mathrm{SINR}),
$$

where $W$ denotes the bandwidth, and the SINR (Signal-toInterference Ratio) is given by:

$$
\mathrm{SINR}=\frac{P g d^{-\alpha}}{N+\sum_{m \in \mathcal{A}} P g d_{m l}^{-\alpha}} .
$$

$P$ denotes the transmission power, $d$ is the distance from the transmitter to the receiver (in meters), $g$ is the gain constant, $N$ is the noise power, $d_{m l}$ denotes the distance from the source of link $m$ to the destination of link $l$, and finally $\alpha$ is the path loss exponent.

\section{MAC protocols - Strong vs. weak interferers}

Transmitters access the channel in a distributed manner using some random multi-access algorithm. We consider CSMA protocols only, where transmitters can start transmitting only when they sense that the channel is idle. The performance analysis is general enough to capture the characteristics of a large class of such algorithms, from the simple non-adaptive ALOHA scheme to more complicated scheme such as the DCF implemented in IEEE 802.11-based networks.

In certain cases, the MAC protocol may try to reserve the channel for its transmission, for example using the RTS/CTS signaling procedure in IEEE 802.11. When link $l$ reserves the channel, neighboring links have to remain inactive. These links are termed strong interferers for link $l$. We denote by $\mathcal{L}_{s t}^{l}$ the set of these links. Of course this set is a subset of $\mathcal{L}_{c(l)}$ the set of links that use channel $c(l)$. If a link operating on channel $c(l)$ is not strongly interfering with link $l$, we say that it is a weak interferer. We denote by $\mathcal{L}_{w}^{l}=\mathcal{L}_{c(l)} \backslash \mathcal{L}_{s t}^{l}$ the set of weak interferers of link $l$. The network performance strongly depends on the definition of the sets of strong interferers, therefore the transmission range of the signaling procedure should be optimized. In Section IV, we propose a way of defining these sets so as to maximize the network capacity. 


\section{Traffic characteristics}

We assume that a fixed number of TCP connections share the resources of the network. These connections (or data flows) are categorized according to not only the set of resources needed to transport the corresponding packets, but also TCP parameters and other external constraints such as the access rate. We consider $K$ classes of flows where a class defined by the route taken by the corresponding packets (we assume fixed and predefined routing here). Denote by $r_{k}$ the route of flows of class $k$. We assume that data packets and TCP acks visit the same set of nodes, which means that $l \in r_{k}$ if and only if the TCP acks go through link $l^{-}$. Then, $r_{k}^{-}$defines the set of links used by TCP acks of flows of class $k$. A given link $l$ can carry either data packets of length $\sigma$ or TCP acks of size $\sigma_{\text {ack }}$. We denote by $\beta$ the ratio of these two sizes, $\beta=\sigma_{\text {ack }} / \sigma$.

In the following we denote by $x=\left(x_{1}, \ldots, x_{K}\right)$ the vector representing the numbers of flows of each class. Finally we denote by $\mathcal{L}(x)$ the set of links that are needed when the network state is $x, \mathcal{L}(x)=\cup_{k: x_{k}>0}\left(r_{k} \cup r_{k}^{-}\right)$.

\section{E. Rate region}

Informally speaking the rate region refers to the set of rates at which the various data flows can be simultaneously transmitted. Data flows of the same class should receive the same throughput, so it is useful to define the rate region as the set of achievable vectors $\phi=\left(\phi_{1}, \ldots, \phi_{K}\right)$ representing the total rates at which the flows of the various classes are served. The rate region in state $x$ will be denoted by $\mathcal{R}(x)$. For all state $x$, it is a subset of $\mathbb{R}_{+}^{K}$, with, by convention $\phi_{k}=0$ if $x_{k}=0$ for all $\phi \in \mathcal{R}(x)$. In the following we will denote by $\mathcal{R}_{\mathcal{K}}$ the rate region when the set of active flow classes (the set of classes $k$ such that $x_{k}>0$ ) is $\mathcal{K}$. The vector $\phi$ belongs to the rate region if it corresponds to a feasible packet level behavior of the network. By feasible, we mean that:

(i) all buffers in the network must remain stable,

(ii) $\phi$ is greedy in the sense that at least one buffer in the route of each active flow is saturated.

Note that by definition, the set of buffers of a given route is composed of buffers handling actual packets from the source to the destination, but also by buffers handling the acknowledgments of the congestion control layer. The second condition (ii) is a consequence of the greedy behavior of the congestion control protocol: if a flow does not saturate at least one link on its route, the protocol will increase the sending rate.

\section{AN APPROXIMATION OF THE RATE REGION}

Characterizing the rate region of data networks is generally very challenging, even in the case of wired networks where the link capacities are fixed (we have a network of queues with source routing whose stability region is generally unknown). However for most routing schemes, it can be conjectured that the rate region is actually the set of vectors $\phi$ such the traffic going through each link remains smaller than the capacity of the link. In the case of wireless networks with distributed
MAC, characterizing the rate region is even more difficult, for the following reasons:

(a) The capacity of a link is not well-defined as it depends on the activity of other links through strong and weak interference. Then at the packet level, the network behaves as a system of interacting queues whose stability is largely unknown [15]-[17], although there have been recent promising progresses [18].

(b) An additional difficulty is introduced when the behavior of congestion control algorithms must be taken into account. Indeed, since the link capacities depend on the activity and hence the buffer content of interfering links, the fact that the congestion control algorithms tend to saturate at least one buffer on each route, impacts the link capacities.

(c) The behavior of distributed adaptive MAC protocols proves extremely difficult to analyze. A rigorous analysis, for example for the DCF, would have to include an analysis of the behavior of the multi-dimensional Markov chains representing the evolution of the back-off windows of the various transmitters. This proves to be intractable in general.

To solve the above difficulties, we propose a heuristic based on decoupling arguments similar to those used by Bianchi in his analysis of WLAN hotspots [19], and on recent averaging arguments proposed by Marbach et al. [20].

We now explain the main ideas behind the proposed rate region approximation. In order to determine how much traffic a link $l$ can handle, we must (1) characterize the epochs at which the corresponding transmitter could actually start transmitting (recall that we consider CSMA protocols); (2) evaluate the probability with which it actually transmits at these epochs; and (3) finally account for the impact of weak interferers on the link rates. A rigorous analysis of problem (1) would involve the study of a loss network as performed in [21] for example, and in general, the epochs at which strong interferers can start transmitting do not coincide. As a result, solving problem (2) in case of adaptive MAC protocols becomes extremely challenging. In [22], a rigorous treatment of both (1) and (2) is proposed. However, it cannot lead to explicit formulas for the link throughputs. In certain scenarios, the two problems can be solved approximately assuming that when a transmitter for link $l$ is allowed to transmit, its strong neighbors are also allowed to transmit. This is the case when either all links of $\mathcal{L}_{s t}^{l}$ interfere with each other, or in case of certain large networks under the primary interference model as explained in [20]. In the latter paper, the authors use approximation results for large loss networks to justify the simplification. In the following, we use the approximation that has just been described to predict the throughput of links.

It is important to note that the approximation does not hold for all mesh wireless networks. In multi-channel networks such those based on IEEE 802.11a, channels will be assigned such that links using the same channel are not very close and the only strong interferer in most cases is the opposite direction of 
the same link. In such networks, the proposed approximation is then justified.

\section{A. Transmission probabilities}

For adaptive MAC protocols, the stationary probability $p_{l}$ at which link $l$ attempts to use the channel can be well approximated by applying the decoupling approach as in [19]. This approach assumes that in order to compute the probability $p_{l}$, we can consider that the behavior of link $l$ depends on that of the strongly interfering links only through a constant stationary collision probability $c_{l}$. This assumption then provides a formula relating $p_{l}$ and $c_{l}$ :

$$
p_{l}=F_{l}\left(c_{l}\right) \text {. }
$$

For non-adaptive ALOHA-type algorithms, the functions $F_{l}$ are constants. For IEEE 802.11 algorithms such as the DCF, Bianchi has identified this function: for all links $l$,

$$
F_{l}(c)=\frac{2(1-2 c)}{(C W+1)(1-2 c)+C W \times c\left(1-(2 c)^{n}\right)},
$$

where $C W$ (resp. $C W 2^{n}$ ) is the minimum (maximum) backoff window size in DCF.

For any link $m \in \mathcal{L}_{s t}^{l}$, let $a_{m}$ denote the proportion of time link $m$ is active (it has a packet to send at the instants of the point process discussed above). Then the collision probability for link $l$ is given by:

$$
c_{l}=1-\prod_{m \in \mathcal{L}(x) \cap \mathcal{L}_{s t}^{l} \backslash\{l\}}\left(1-a_{m} p_{m}\right) .
$$

\section{B. Link throughputs}

To compute the throughput of a link, weak interference has to be taken into account. Recall that when the links of a set $\mathcal{A} \subset \mathcal{L}_{w}^{l}$ are transmitting, the rate at which link $l$ may transmit is denoted by $R_{l, \mathcal{A}}$. We can deduce the ergodic average of a packet transmission duration (including physical preamble, and the overhead generated by the MAC protocol) on link $l$ as follows:

$$
\begin{aligned}
& \quad E\left[D_{l}\right]=h_{1}+\left(E\left[\sigma_{l}\right]+a c k\right) \\
& \times \sum_{\mathcal{A} \subset \mathcal{L}(x) \cap \mathcal{L}_{w}^{l}} \frac{1}{R_{l, \mathcal{A}}} \prod_{m \in \mathcal{A}} A_{m} \prod_{n \notin \mathcal{A}, n \in \mathcal{L}(x) \cap \mathcal{L}_{w}^{l}}\left(1-A_{n}\right),
\end{aligned}
$$

where $A_{m}$ denotes the proportion of time link $m$ is transmitting and $E\left[\sigma_{l}\right]$ is the average packet size given as follows:

$$
E\left[\sigma_{l}\right]=\frac{\sum_{k: l \in r_{k}} \phi_{k} \sigma+\sum_{k: l \in r_{k}^{-}} \phi_{k} \sigma_{\text {ack }}}{\sum_{k: l \in r_{k}} \phi_{k}+\sum_{k: l \in r_{k}^{-}} \phi_{k}} .
$$

In (3), $h_{1}$ is a constant representing the overhead at the PHY and MAC layers corresponding to a packet transmission, e.g., using the IEEE 802.11 DCF without RTS/CTS, $h_{1}=$ $\mathrm{DIFS}+\mathrm{SIFS}+2 t^{\mathrm{pr}}\left(t^{\mathrm{pr}}\right.$ denotes the PHY preamble), and $a c k$ denotes the size of the MAC acknowledgment. Note that (3) is obtained by using the decoupling argument (we assume that link activities are independent), and by evaluating the ergodic mean time needed to transmit one bit.
The proportion of time link $m$ is transmitting is given by:

$$
A_{m}=a_{m} \times \frac{p_{m}\left(E\left[D_{m}\right]-h_{2}\right)}{E\left[S_{m}\right]},
$$

where $h_{2}$ is a constant to account for the time a node is not transmitting during $E\left[D_{m}\right]$, e.g., using the DCF without RTS/CTS, $h_{2}=$ DIFS + SIFS. $E\left[S_{m}\right]$ is the mean duration of a slot in $\mathcal{L}_{s t}^{m}$. Here, slot is to be understood as in Bianchi's analysis. A slot can be either an empty slot of duration SLOT, or a successful packet transmission, or a collision. To evaluate $E\left[S_{m}\right]$ we need to compute the average duration of a collision in $\mathcal{L}_{s t}^{m}$. Denote by $E\left[D_{m}^{c o l}\right]$ this duration. In general, it has a complicated expression since we need to carefully account for the duration of transmission of all packets involved in the collision. An exact expression has been given in [14]. In Section III-D, we provide the exact expression for a specific case. We have:

$$
\begin{aligned}
E\left[S_{m}\right]= & \sum_{n \in \mathcal{L}(x) \cap \mathcal{L}_{s t}^{m}} a_{n} p_{n}\left(\left(1-c_{n}\right) E\left[D_{n}\right]\right. \\
& +\prod_{n \in \mathcal{L}(x) \cap \mathcal{L}_{s t}^{m}}\left(1-a_{n} p_{n}\right) \mathrm{SLOT}+p_{m}^{c o l} E\left[D_{m}^{c o l}\right]
\end{aligned}
$$

and

$p_{m}^{c o l}=1-\sum_{n \in \mathcal{L}(x) \cap \mathcal{L}_{s t}^{m}} a_{n} p_{n}\left(1-c_{n}\right)-\prod_{n \in \mathcal{L}(x) \cap \mathcal{L}_{s t}^{m}}\left(1-a_{n} p_{n}\right)$.

Now the throughput of link $l \in \mathcal{L}(x)$ in state $x$ is given by:

$$
T_{l}(x)=\frac{a_{l} p_{l}\left(1-c_{l}\right) E\left[\sigma_{l}\right]}{E\left[S_{l}\right]}
$$

\section{Rate regions}

We are now ready to characterize the rate region in each network state $x$. If flows of class $k$ send traffic at rate $\phi_{k}$ for all $k$ such that $x_{k}>0$, then in the case of network stability, flow conservation provides additional conditions on the traffic of data packets and TCP acks:

$$
\sum_{k: l \in r_{k}, x_{k}>0} \phi_{k}+\sum_{k: l \in r_{k}^{-}} \beta \phi_{k}=T_{l}(x), \quad \forall l \in \mathcal{L}(x) .
$$

The network can then be stable at packet level only if the set of equations (1)-(5) has a solution $\left\{\left(a_{l}, p_{l}, c_{l}\right), l \in\right.$ $\mathcal{L}(x)\}$, such that for all $l, a_{l}, p_{l}, c_{l} \in[0: 1]$. Note that the existence and uniqueness of such solution can be analyzed using asymptotic analysis when the network is large [22]. In addition, accounting for the greedy behavior of the congestion control algorithm, condition (ii) must hold. In summary, the vector of traffic $\phi$ is feasible if:

$$
\begin{gathered}
\forall l \in \mathcal{L}(x), \exists p_{l}, a_{l}, c_{l} \in[0: 1] \text { satisfying }(1)-(5), \\
\forall k: x_{k}>0, \exists l \in r_{k} \cup r_{k}^{-}, a_{l}=1 .
\end{gathered}
$$

Finally the rate region in state $x$ is given by the following proposition that summarizes the analysis presented above.

Proposition 1: The rate region when the numbers of flows of each class is $x$ is given by:

$$
\mathcal{R}(x)=\{\phi:(7),(8) \text { are satisfied }\} .
$$




\section{Self interference and downloads only}

In this subsection, we illustrate the above analysis in a relevant example where the channel allocation is made so as to reduce strong interference, with the only strong interferer for link $l$ being link $l^{-}, \mathcal{L}_{s t}^{l}=\left\{l^{-}\right\}$. We also assume that routes to the nodes connected to the Internet are all in a given direction. This implies that in some route $r_{k}$, each link $l$ transports only data packets, and link $l^{-}$carries only TCP acks. An example of such networks is presented and numerically analyzed in Section V. In that case the model is slightly simplified. We have, for all $k$ such that $x_{k}>0$, for all $l \in r_{k}, p_{l}=p_{l^{-}}$, $c_{l}=c_{l^{-}}, a_{l}=a_{l^{-}}, E\left[\sigma_{l}\right]=\sigma, E\left[\sigma_{l^{-}}\right]=\sigma_{\text {ack. }}$. The mean duration of a slot in $\mathcal{L}_{s t}^{l}$ has the following expression:

$$
\begin{aligned}
E\left[S_{l}\right]= & a_{l} p_{l}\left(1-a_{l} p_{l}\right)\left(E\left[D_{l^{-}}\right]+E\left[D_{l}\right]\right) \\
& +\left(1-a_{l} p_{l}\right)\left(1-a_{l} p_{l}\right) \mathrm{SLOT}+\left(a_{l} p_{l}\right)^{2} E\left[D_{l}\right] .
\end{aligned}
$$

Note that the mean duration of a collision is always equal to $E\left[D_{l}\right]$, the mean duration of the longest packet. Finally the link throughputs are related to the source sending rates by:

$$
\sum_{k: l \in r_{k}, x_{k}>0} \phi_{k}=T_{l}(x) .
$$

We do not need to compute $T_{l^{-}}(x)$ since obviously, $T_{l^{-}}(x)=$ $\beta T_{l}(x)$.

\section{TOWARDS OPTIMAL INTERFERENCE MANAGEMENT}

Based on the performance model derived in the previous section, we may address several important questions arising when designing mesh networks. Here we discuss how our model can be used for interference management. We assume in the following that when a transmitter attempts to use a channel, it does so with full power. This principle simplifies the radio resource management, and has been shown to be nearly optimal [13]. It then remains to define for a given link, which links in its neighborhood can be activated simultaneously. We also assume that each transmitter runs the DCF algorithm corresponding to the IEEE 802.11 standards.

\section{A. Strong and weak interference}

The RTS/CTS signaling mechanism was designed to avoid collisions between simultaneous transmissions, with collision implying that the interference from such transmissions is so high that the receiver cannot decode the packet. Such strong interference can be combated by a properly designed contention resolution algorithm. For IEEE 802.11-based networks where access to the channel is distributed, this means that strong interferers (using the same channel) must be silenced when a given node wants to transmit. This is achieved by RTS/CTS signaling.

Links that use the same channel as a given link under consideration, yet are far away as to cause only weak interference, may not need to be silenced. If the interference is weak enough, advanced coding mechanisms such incremental redundancy may be used to combat this interference through rate control. It remains to determine how interferers are classified as strong or weak as we do in the next subsection.

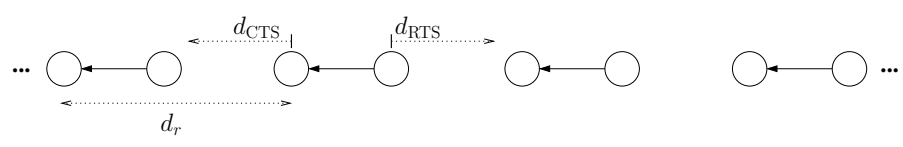

Fig. 1. A linear network.

\section{B. Design of the RTS/CTS range}

The objective of the RTS/CTS signalling procedure is to exclude links that interfere strongly with a given transmission, while allowing weak interferers to transmit simultaneously. Nodes within the RTS/CTS signalling coverage range are classified here as strong interferers and the rest are weak interferers. If this range is too large, then a link must share transmission time with many other links, thus reducing perlink throughput. A small range on the other hand, will reduce the transmission rate due to interference resulting from a large number of weak interferers (by reducing the SINR). Here we examine this tradeoff and using the performance model presented in Section II-E, we define the optimal range for an illustrative example of the regular symmetric network depicted in Figure 1. We define $d_{r}$ to be distance between receivers whose links use the same channel. This parameter may be interpreted as a measure of channel reuse, the reuse being high for small values of $d_{r}$. We also denote $d_{\mathrm{RTS}}$ and $d_{\mathrm{CTS}}$ to be the range of RTS/CTS signaling from the transmitter and receiver respectively. We assume the two distances to be of equal value, and use $d_{\text {RTS }}$ to represent both values. Note that under this signalling procedure, all nodes at a distance of $d_{\text {RTS }}$ from the transmitter or receiver must be silent for the subsequent transmission. For a given $d_{r}$ then, we find the range $d_{\text {RTS }}$ such that the link throughput as calculated using (5) is optimal. For this illustrative example, we consider the link length, the distance for transmitter to receiver, to be $45 \mathrm{~m}$. We start with $d_{r}=45 \mathrm{~m}$, which represents the case of full channel reuse, and consider values of increasing $d_{r}$. We observed that in the case of full channel reuse, it is optimal to silence the two closest interferers on each side. For $45<d_{r}<85$ the optimal range of $d_{\mathrm{RTS}}$ is up to the closest interferer on each side. For $d_{r} \geq 85$, the link throughput is optimal when no links using the same channel are silenced.

The approach followed above to distinguish between weak and strong interferers of a given link (and thus set the RTS/CTS range in IEEE 802.11 networks) can be applied to more general networks in a similar manner. As it turns out, the optimal coverage range is much smaller than that used by default in equipments today. This means that when ideal and fast rate control is used (which is not the case today), the optimal RTS/CTS range will need to be re-defined.

\section{NumERICAL EXAMPLE: A TREE NETWORK}

We now apply our model to an example of mesh networks in order to illustrate the theoretical results of Section II-E. We consider the network presented in Figure 2. It has a tree topology with the root having a high speed wired connection to the Internet, and with leaves being WLAN Access Points (AP). 
Three classes of flows compete for the use of the resources. We assume that only five channels are available for this network. A single channel is allocated to each link and the corresponding reverse link. Each node implement DCF.

Using the results of the previous section, it can be shown that with the channel assignment presented in Figure 2, it is optimal that the set set of strong interferers for each link reduces to the reverse link. In other words, the obtained network satisfies the conditions of Section III-D. For numerical experiments, in order to match the radio parameters of networks based on the IEEE 802.11a standard, we choose a bandwidth $W$ equal to $22 \mathrm{MHz}$, a maximum power ranging from $100 \mathrm{~mW}$ to $800 \mathrm{~mW}$ depending on the legislation in various countries, a noise power equal to $-130 \mathrm{~dB}, g$ equal to $-55 \mathrm{~dB}$, and a path loss exponent $\alpha$ of 3.5 .

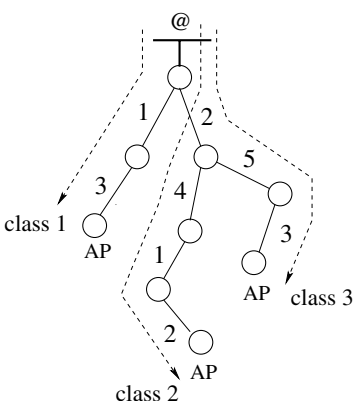

Fig. 2. An example of a multi-channel mesh network. The links are identified by the corresponding channel assigned.

The rate regions corresponding to this network are presented in Figure 3. Recall that $\mathcal{R}_{\mathcal{K}}$ denotes the rate region of the network when the set of active flow classes is $\mathcal{K}$. The results are obtained when the link lengths are all equal to $45 \mathrm{~m}$. The data packet size is 1000 bytes. Note that the serving rate of flows of a given class does not exceed $15.7 \mathrm{Mb} / \mathrm{s}$ (this corresponds to a PHY rate of $54 \mathrm{Mb} / \mathrm{s}$ ), due to the overhead induced by the MAC protocol and due to the presence of TCP acks. Also observe that weak interference is quite low even if the network seems quite compact.

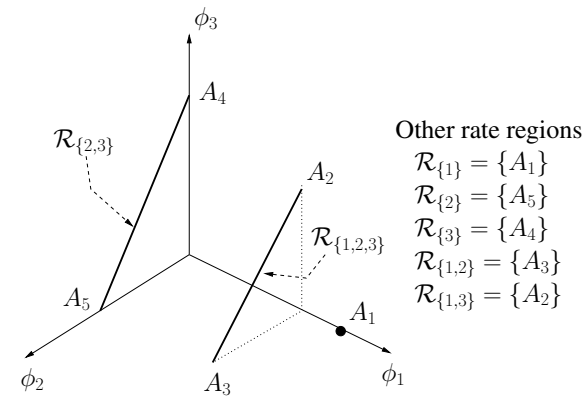

Fig. 3. The various rate regions corresponding to the tree network of Figure $2-A_{1}=(15.7,0,0), A_{2}=(13.2,0,12.8), A_{3}=(13.2,13.4,0)$, $A_{4}=(0,0,15.7), A_{5}=(0,15.7,0)$, all values are in $\mathrm{Mb} / \mathrm{s}$.

\section{REFERENCES}

[1] J. Eriksson, S. Agarwal, P. Bahl, and J. Padhye, "Feasibility study of mesh networks for all-wireless offices," in Proc ACM MobiSys'06, 2006.

[2] M. Alicherry, R. Bhatia, and E. L. Li, "Joint channel assignment and routing for throughput optimization in multi-radio wireless mesh networks," in Proc. ACM MobiCom, 2005.

[3] P. Kyasanur, J. So, C. Chereddi, and N. H. Vaidya, "Multi-channel mesh networks: Challenges and protocols," IEEE Trans. Wireless Commun., vol. April, 2006.

[4] IEEE Std 802.11, "IEEE Standard for Wireless LAN Medium Access Protocol and Physical Layer Specifications," Aug. 1999.

[5] IEEE Std 802.16, "Part 16: Air Interface for Fixed Broadband Wireless Access Systems," July 2004.

[6] A. Adya, P. Bahl, J. Padhye, A. Wolman, and L. Zhou, "A multi-radio unification protocol for IEEE 802.11 wireless networks," in Proc. IEEE BroadNets, 2004

[7] A. Raniwala and T. Chiueh, "Architecture and algorithms for an IEEE 802.11-based multi-channel wireless mesh networks," in Proc. IEEE Infocom, 2005.

[8] P. Kyasanur and N. H. Vaidya, "Routing and interface assignment in multi-channel multi-interface wireless networks," in Proc. IEEE WCNC, 2005.

[9] — - "Routing and link-layer protocols for multi-channel multiinterface ad hoc wireless networks," SIGMOBILE Mobile Computing and Communications Review, vol. 10, no. 1, pp. 31-43, Jan. 2006.

[10] M. Kodialam and T. Nandagopal, "Characterizing achievable rates in multi-hop wireless networks: The joint routing and scheduling problem," in Proc. ACM MobiCom, San Diego, CA, USA, 2003.

[11] _ "Characterizing achievable rates in multi-hop wireless with orthogonal channels," IEEE trans. on Networking, vol. 13, no. 4, pp. 868$880,2005$.

[12] A. Giannoulis, K. Tsoukatos, and L. Tassiulas, "Lightweight cross-layer algorithms for fairness and energy efficiency in cdma ad-hoc networks," in Proc. IEEE WiOpt, 2006.

[13] B. Radunovic and J.-Y. L. Boudec, "Rate performance objectives of multihop wireless networks," IEEE Transactions on Mobile Computing, vol. 3, no. 4, 2004.

[14] F. Lebeugle and A. Proutière, "User-level performance of WLAN hotspots," in Proc. ITC 19, Apr. 2005.

[15] W. Szpankowski, "Stability conditions for some multiqueue distributed systems: Buffered random access systems," Adv. Appl. Probab., vol. 26 , pp. 498-515, 1994.

[16] W. Luo and A. Ephremides, "Stability of $n$ interacting queues in random-access systems," IEEE trans. on Information Theory, vol. 45, no. 5, pp. 1579-1587, 1999.

[17] T. Bonald, S. Borst, N. Hegde, and A. Proutiere, "Wireless data performance in multi-cell scenarios," in Proc. ACM Sigmetrics, 2004.

[18] C. Bordenave, D. McDonald, and A. Proutière, "Random multi-access algorithms, an asymptotic approach," in Proceedings of ACM Sigmetrics, 2008.

[19] G. Bianchi, "Performance analysis of the IEEE 802.11 distributed coordination function," IEEE J. Select. Areas Commun., vol. 18, no. 3, pp. 535-547, 2000.

[20] P. Marbach, A. Eryilmaz, and A. Ozdaglar, "Achievable rate region of CSMA schedulers in wireless networks with primary interference constraints," in Proceedings of IEEE Conf. on Decision and Control, 2007.

[21] M. Durvy and P. Thiran, "Packing approach to compare slotted and non-slotted medium access control," in Proceedings of IEEE Infocom, 2006.

[22] C. Bordenave, D. McDonald, and A. Proutière, "A particle system in interaction with a rapidly varying environment: Mean field limits and applications," submitted to Stochastic Processes and their Applications, 2007. 